\title{
Multiple sclerosis plaque related to abnormal somatosensory evoked potentials
}

\author{
W. B. MATTHEWS AND MARGARET ESIRI \\ From the University Department of Clinical Neurology, Churchill Hospital, and \\ the Department of Neuropathology, Radcliffe Infirmary, Oxford
}

SUMMARY A patient with mild multiple sclerosis died from a ruptured intracranial aneurysm. It was possible to relate abnormalities of somatosensory evoked potentials recorded some months earlier to a plaque involving the root entry zone in the cervical spinal cord which had not resulted in clinical sensory abnormalities.

Averaged evoked potential techniques are being used increasingly in the diagnosis of multiple sclerosis. The underlying assumption is that persistent abnormalities of evoked potentials, in the absence of relevant clinical symptoms or signs, are evidence of persistent structural lesions of the central nervous system. Opportunities to verify this assumption must necessarily be rare and arise by chance. Death in multiple sclerosis usually occurs after a prolonged period of severe disability during which investigations are seldom performed. The identification at necropsy of symptomless plaques in such patients would in any case be impossible. No anatomical verification of the relationship of a multiple sclerosis plaque to abnormalities of somatosensory evoked potentials (SEP) has been published. A patient is described in whom death from other causes while mildly affected by multiple sclerosis allowed this relationship to be established.

\section{Case report}

The symptoms of this woman (CV) began in 1967 at the age of 39 years when she developed retrobulbar neuritis of the left eye and tingling in the fingers of both hands followed by recovery within a few months. In 1968 vision of the left eye was again affected, a central scotoma being documented on hospital attendance. In 1969 she developed paraesthesiae in the right foot and was found to have incoordination of the right lower

Address for reprint requests: Professor W. B. Mat thews, Department of Clinical Neurology, Churchill Hospital, Headington, Oxford OX3 7LJ.

Accepted 17 April 1979 limb but no other signs. This again recovered, but in 1970 there was a transient episode of weakness of the left leg. Remission persisted until March 1974 when the left leg again became weak, on this occasion with incomplete recovery.

She first attended the Radcliffe Infirmary in February 1977 having sustained a further episode of retrobulbar neuritis affecting the right eye in December 1976. Visual acuity was $\mathrm{R} 6 / 5$, L 6/12O Both optic discs were pale. There was no nystage mus. She had no symptoms or abnormal signs ine the upper limbs and, in particular, there was no sensory loss to stringent clinical examination? There was generalised weakness of the left lower limb with increased tendon reflexes and an extensor plantar reflex but again no sensory loss.

Visual evoked potentials (VEP) and SEP were examined on 21 March 1977. No measurable VEP could be recorded on stimulation of either eye with an alternating checkerboard pattern. A normal cervical SEP was recorded on stimulation of the right median nerve (Matthews et al., 1974; Small et al., 1978). On stimulation of the left median nerve the normal pattern of $\mathrm{N} \overline{11}, \overline{13}$, and $\overline{14}$ peaks (Jones, 1977) could not be recognised (Fig. 1). An early cortical wave (N20) was recorded with a latency of $23 \mathrm{~ms}$ on stimulation of the right median nerve but no early potential could be evoked from the left median nerve.

When seen in May 1977 the patient had experienced no fresh symptoms. On 25 October she was admitted in coma having sustained a subarachnoid haemorrhage. Decerebrate rigidity developed, and she died two days later. A necropsy was performed. The brain was sliced coronally after fixation. There was a saccular aneurysm on 40 

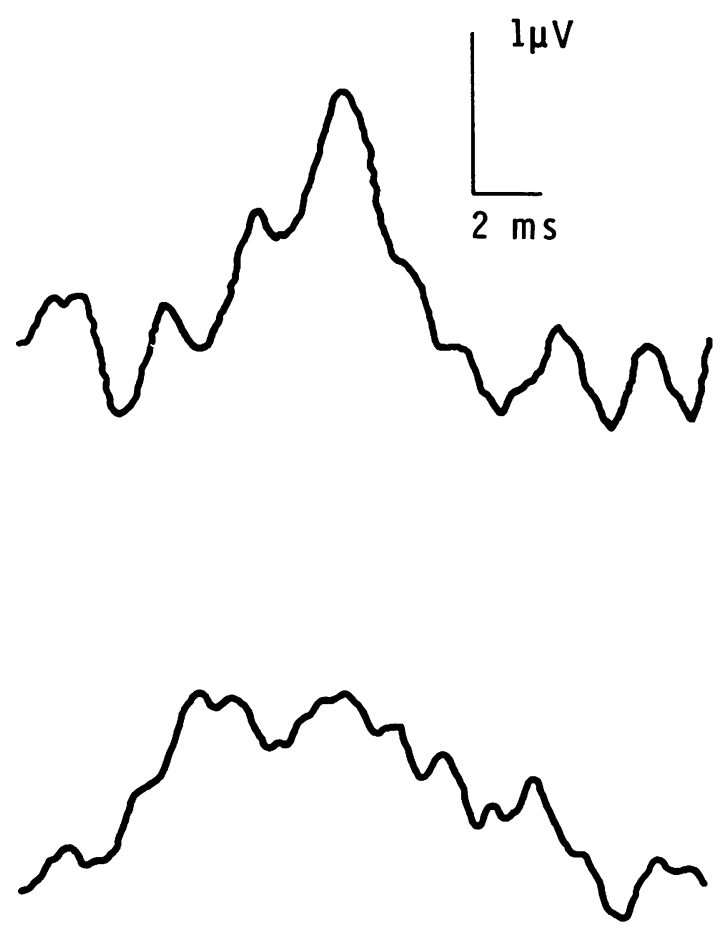

Fig. 1. Recording from the skin over the sixth cervical intervertebral space with a midfrontal reference electrode after stimulation of the right (upper trace) and left (lower trace) median nerves: 250 potentials are averaged. The potential evoked from the right median nerve is normal but there is no recognisable pattern evoked from the left median nerve.

the left middle cerebral artery which had ruptured and caused subarachnoid and massive intracerebral haemorrhage.

The intracranial portions of both optic nerves and the spinal cord at multiple levels were examined in transverse sections stained with haematoxylin and eosin, luxol fast blue/cresyl violet, the Palmgren stain for axons, and the Holzer stain for glial fibres. In the cervical region of the spinal cord sections were examined from C2-T1 segments at $1.0 \mathrm{~mm}$ intervals. Semiserial sections at approximately $1.0 \mathrm{~mm}$ intervals of the medulla were also examined. The pons was examined at three levels, the midbrain at two levels, and the right thalamus with semiserial sections at $1.0 \mathrm{~mm}$ intervals.

The intracranial portion of the left optic nerve contained a demyelinated plaque involving almost all the nerve fibres at one level (Fig. 2). Axons appeared well preserved. The intracranial portion of the right optic nerve contained no plaques. The

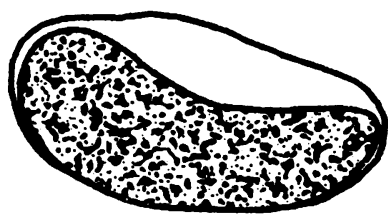

Fig. 2. Camera lucida drawing of transverse section of left optic nerve stained for myelin. The extensive area of demyelination is indicated by shading.

intraorbital portions of both nerves were not available for study. The optic chiasm, optic tracts, and optic radiations contained no plaques that were visible macroscopically, or in representative sections but no attempt was made to section these structures serially.

In the spinal cord an extensive plaque was present in the left cervical region extending from C3 to T1 segments. It occupied the major part of the lateral column of white matter with extensions involving the dorsal root entry zone and the most lateral fibres of the left posterior column at two levels, C3/4 and C8/T1 segments (Fig. 3). Smaller plaques were also present in the cervical region (Fig. 3), and a few plaques in thoracic and lumbar regions but these were all less than $5 \mathrm{~mm}$ diameter and none of them involved the posterior columns on either side.

Within the medulla the only plaque found was one measuring no more than $1.0 \mathrm{~mm}$ in any direction in the left pyramidal tract. The posterior column nuclei showed no demyelination. Within the pons there were plaques measuring up to $3 \mathrm{~mm}$ diameter in the root of the right middle cerebellar peduncle, right and left superior cerebellar peduncles and on the inferior surface of the basis pontis on the right. None of these plaques involved sensory fibres. The midbrain and right thalamus contained no plaques.

All the plaques showed evidence of gliosis and had the histological appearances of chronic lesions. Axons appeared relatively well preserved.

\section{Discussion}

In the absence of histological evidence of fresh plaque formation or of clinical evidence of recent relapse it is reasonable to believe that the plaques found at necropsy were those present at the time of recording the evoked potentials seven months earlier. It was not possible to relate visual function and abnormalities of VEP with the anatomical lesions because the intraorbital portions of the optic nerves were not examined.

The abnormality of short latency SEP can, 
C3

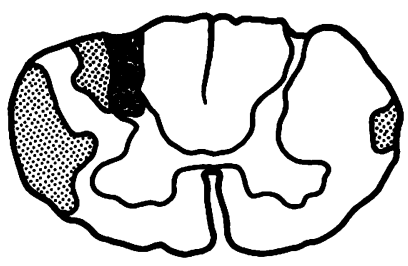

C8

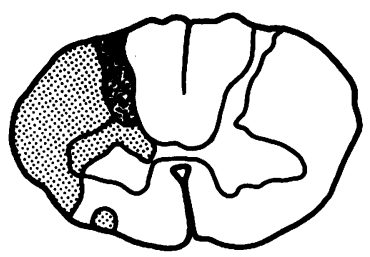

C4

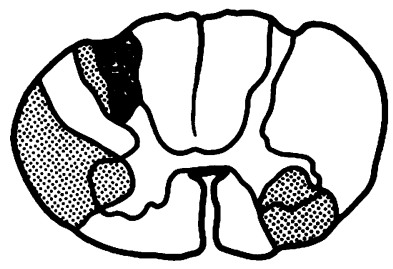

Tl

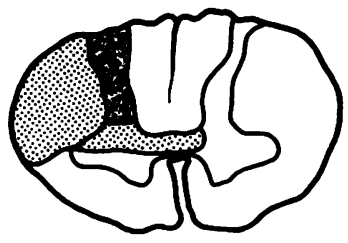

Fig. 3. Camera lucida drawings of spinal cord transverse sections stained for myelin. Areas of demyelination are shaded with accentuation of shading to show areas involving the dorsal root entry zones and lateral part of the left posterior column.

however, clearly be attributed to the plaque in the cervical spinal cord involving the root entry zone and lateral aspect of the posterior column on the appropriate side and at the appropriate level. This finding cannot throw any light on the source of subcortical SEP, whether spinal cord or more rostral structures (Wiederholt, 1978), but confirms that the method can detect clinically silent plaques in the sensory pathways. The plaque was clearly responsible for the spastic weakness of the left lower limb but had caused no sensory symptoms or abnormal signs in the upper limb with the possible exception of tingling in the hands 10 years earlier. The basic assumption underlying the diagnostic use of somatosensory evoked potentials in multiple sclerosis is thus confirmed.

References

Jones, S. J. (1977). Short latency potentials recorde from the neck and scalp following median nerv $\mathbb{2}$ stimulation in man. Electroencephalography an $\Phi^{\circ}$ Clinical Neurophysiology, 43, 853-863.

Matthews, W. B., Beauchamp, M., and Small, D. O (1974). Cervical somatosensory evoked responses if man. Nature, 252, 230-232.

Small, D. G., Matthews, W. B., and Small, M. (1978递 The cervical somatosensory evoked potential (SEPF in the diagnosis of multiple sclerosis. Journal of 6 the Neurological Sciences, 35, 211-224.

Wiederholt, W. C. (1978). Recovery function of short latency components of surface and depth recorded somatosensory potentials in the cat. Electroencepha lography and Clinical Neurophysiology, 45, 259-267. 